\title{
Intelligent Agent Based RFID System for on Demand Bus Scheduling and Ticketing
}

\author{
Paul Hamilton and Suresh Sankaranarayanan
}

\begin{abstract}
The public transportation system needs some change for satisfying the commuting needs of the general public. These systems are operated by state run authorities or by private parties. The commuters face challenges as far as accessing the public transportation facility as needed. This is mostly attributed to the static scheduling of bus trips, within some pattern, regardless of fluctuations in commuters demand. This is very common in many countries and Jamaica is not exception in this respect. There has been quite an amount of research work carried out in using Radio Frequency Identification (RFID) technology in public transportation system towards tracking of passengers while they board and exit the bus. Research has also been carried out on tracking passengers possessing RFID enabled Smart card and store in central locations for future viewing if need be, which a privacy concern is really. Also research has been carried out in using RFID technology towards tracking of buses by deploying RFID sensors at traffic stop lights, intersections, etc., for updating bus arrival time which user can view in his cell phone. In addition research is being conducted in using mobile agents with RFID technology towards passenger demand and carrying capacity. It is therefore clear from literature available on the subject that there has been no work reported so far in dynamic scheduling of buses based on passenger demand by means of RFID usage. So our proposed research work would be on dynamic scheduling of buses from the point of view of passenger tracking pattern, which would be noted and included in planning and scheduling by means of intelligent agents.
\end{abstract}

Index Terms-RFID, agents, commuters, MATSim.

\section{INTRODUCTION}

The emergence of Radio Frequency Identification (RFID) Technology has been heralded as a prime solution to the problem of real-time tracking in the transportation arena. It is based on automatic identification technology that enables non-contact reading of data, thus making it ideal for applications in manufacturing, warehousing, logistics, retail, healthcare and in public transportation to name a few. RFID Technology is being viewed as the way forward thus gradually moving away from the traditional Bar Code system for the identification of items. RFID's versatility is proven more dominant due to the fact that it is a non-line-of sight technology which does not require items to be handled manually for reading as RFID readers are capable of reading tags even when they are hidden. The application of RFID

Manuscript received December 19, 2012; revised February 23, 2013.

Paul. Hamilton is with the Department of computing, University of West Indies, Jamaica (e-mail: hamilton.pablo@gmail.com)

Suresh. Sankaranarayanan is with Computing and Information Systems, Institut Teknologi Brunei, Brunei \& Visiting Professor, Department of Computing, University of WestIndies, Jamaica (e-mail: pessuresh@hotmail.com) technology may be enhanced with the use of Mobile Agents, which are compositions of computer software and data which also are able to migrate (move) from one computer to another autonomously and continue its execution on the destination computer. Having hundreds of vehicles in a public transportation system one has to employ numerous resources to keep the system functional and also enable serving the public's needs. However, the efforts of these entities are thwarted by inappropriate trip scheduling, which sees the companies suffering substantial losses and commuters inconvenienced. Without appropriate trip scheduling, there can be many empty buses idly parked at termination points awaiting rigid departure times while potential passengers keep crowding at bus stops due to unforeseen circumstances. This scenario results in having many passengers getting stranded and also a loss for the bus company in the form of wasted fuel and other resources. The running of empty buses when there is low passenger demand foster a bunching of buses at termination points, where buses are parked with engines idling and air conditioning units running needlessly, on account of obeying a static trip schedule which causes starvation at bus stops, thereby losing potential passengers to other forms of transportation.

In addition to inappropriate trip scheduling, the issue of ticketing also bears some passenger inconvenience. This is attributed to the fact that in many countries like in UK, USA, Australia and Jamaica utilize a combined cash and cashless system for payment of fares for the ticket. The current cashless system predominantly seen in many countries using a SmartCard [1] which on placing on the Ticketing machine in bus gets the fare automatically deducted and a ticket issued bearing the amount paid, date and time of the transaction along with the remaining value in the card, which is achieved via the use of an Electronic Ticketing Machine (ETM). The card is rechargeable and can be personalized in case of loss or theft, where it may be disabled if desired. Subsequently, another cashless system was introduced, known as "SmarterCard" [1] which promised improved fare collection and passenger flow, among other benefits. However, not much has been said in terms of reviews pertaining to this system. In the buses presently operating, currently being manned by a single individual playing the dual role of both driver and conductor, lengthy queues are formed to enter the buses resulting from delays caused by the presenting of cards, the collection of cash and making change, to the issuing of tickets.

Taking above issues into consideration, we here propose a smart public transportation system wherein RFID and Agent technology to dynamic bus trip scheduling and an improved cashless payment system have been applied. This application 
will see a complete updating of the technology domain in which public transportation is being scrutinized, by way of facilitating tracking and scheduling of buses along with the monitoring of bus/commuter interaction with emphasis on supply and demand. Being able to display exactly where buses are located at any particular time along with commuter density at stops through RFID tagging, will also greatly enhance scheduling capability. This proposed system is being implemented by utilizing GMap, onto which the coordinates for bus stops, bus indicators and commuter populous are being overlaid. A version of this implementation will be afforded to commuters via an Android application package for mobile devices using JADE-Leap agent development kit, wherein the user may view a map showing his or her current location and that of a desired bus plus the ability to query bus schedules. With regard to commuter frequency, a mobile agent may learn the pattern of the commuters and act accordingly by automatically presenting the user with scheduling updates, fare top-up reminders and desired bus proximity and estimated time of arrival (ETA). RFID enabled smart cards will be ideal in the context, for commuter polling and ticket purchasing due to its ease of use and range. The rest of paper is organized as follows. Section II talks on literature surveyed on RFID usage in public transportation followed by the application of Agent technologies. Section III talks on Agent based RFID Bus Scheduling system which is our proposed work. Section IV talks on the Architectural details and system flow proposed, followed by the developed algorithm for the proposed work. Section V gives the concluding remarks and future work.

\section{LITERATURE SURVEY}

We will survey in brief in this section on the various literatures citing the use of RFID and agent technologies for public transportation usage. It has been expressed that we have the potential passengers passing through a portal in different levels of crowdedness (simulated bus door) carry with them commercial off-the-shelf RFID devices like RFID smart cards and antennas, in an effort to have them detected. The idea expressed enables detecting all passengers as they board and exit the bus. It was an attempt to use the RFID technology effectively for this type of application. However, it should be noted that in cases of no "line of sight" between the devices/smart cards and readers in accordance with the radiation patterns and positioning of antennas, there may be performance fluctuations as these factors are critical to recognition [2].

Research has also been carried out, on the use of pervasive information systems and their acceptance, compared with existing user acceptance theories. This is said to be critical due to the wide variety of system users ranging from novices to experts. However, evaluating acceptance of information systems using existing theories may prove challenging as an individual may use the system without even realizing it, such as arriving at a bus stop and getting scanned via smartcard which results in the required bus being dispatched or fast tracked to that bus stop. Hence a hybrid model is proposed that incorporates established traditional information systems user acceptance theories. To facilitate this, a pervasive system was developed and experiments carried out and evaluated in a laboratory setting. [3]

Research was also focused on how RFID Technology can be used to solve problems faced by public transportation authorities in metropolitan cities by exploring automated tracking of buses that can be used to provide useful estimates of bus arrival times and in turn enhance passenger convenience. However, in any environment of widespread RFID deployment such as a public transit system where RFID transceivers may number in the thousands, there will definitely be challenges faced in the capturing, storage and retrieval of data. As a result, the study also looks at what is involved in collecting data which represent events that took place in the monitored environment and the management of this data which at times can be in large amounts. Such a real time tracking and monitoring system is employed which utilizes an Event, Condition and Action (ECA) framework [4]. This technique aids in efficiently filtering data to remove unwanted or inaccurate instances and then categorizing useful data by aggregation. Also, it is discussed how collected data can be utilized to predict bus movement in an effort to improve the service.

Cards embedded with RFID used in public transportation enables the tracking of individuals with tracking data being stored on a central server. This allows an individual's entire history to be displayed as desired [5]. Research has also been conducted on privacy concerns with respect to, not just the collection of personal information but the aggregation and centralization of personal information.

Research also looked at an Automated Fare Collection (AFC) System also known as the Transit Smart Card System. The advantages of an AFC over a manual fare collection system is highlighted where the AFC is said to lower labor costs and increase efficiency in fare collection and management of this data. The desire to extract more data than just a deduction of fare from transit smart cards, has led to research efforts in extracting data such as points of origin where a passenger would board a bus and have this data recorded as the passengers' smart card is scanned. To achieve this, a Markov chain based Bayesian decision tree algorithm is developed in this study, where the algorithm is verified with the use of public transportation vehicles that are outfitted with GPS tracking and data loggers. Conclusively, it is stated that data collected to represent points of origin when a passengers' transit smart card is scanned, is crucial to the process of transit system planning [1].

In another research application of electronic payments, proxy re-encryption and anonymity to the problem of privacy in public transportation systems that employ electronic ticketing has been mentioned. The architecture proposed encompasses the needs of a typical metropolitan transportation system, preserves the security requirements of the user and the transit company, while enhancing the privacy of the passengers. The incorporation of passive RFID transponders and smart phones enables the use of active devices and allows for more robust security and privacy that surpasses the typical passive RFID transponder architecture [5], [6].

There has also been research conducted on finding a balance between passenger demand and carrying capacity 
within a public transportation system, to facilitate a high quality service at acceptable costs. A proposed Agent-based Micro-simulation is utilized to evaluate decision making at the operational level. The introduction of transit smart card ticketing systems have fostered the growth of agent population, which facilitated an experiment centered around four months of individual mobility data from passengers spanning three(3) modalities in a Dutch public transportation system to generate agent populations using a unique smart card dataset

Due to the vast amounts of detailed data captured by the use of smart cards in a public transportation system, analyzing all of this data to aid in the decision making process is no simple undertaking hence the proposal of an Agent -based Micro-simulation. This simulation sees individual passengers and vehicles being modeled through agents that interact with the public transportation system based on their assigned tasks. In this study, the MATSim simulation package is used to utilize its active user-base. MATSim requires all agents in its populous adjust their plans to improve their effectiveness. Consequently, MATSim will continue running until maximum efficiency is reached within the agent population [7].

Research was also done at the appropriate security and privacy requirements for e-ticketing via RFID technology while highlighting the inadequacy of existing proposals and presenting solutions for privacy-preserving e-tickets based on RFID technology, along with known cryptographic techniques which will help to discourage ticket forgery. E-ticket use is stated to help lower operation and maintenance costs for the transit authority and allow faster and more convenient verification for passengers than paper-based or cash-based payment methods [1], [6].

However, the risks associated with e-ticketing, is the use of spatio-temporal data for the authentication of tickets. It is of utmost importance that this information is not privy to unauthorized parties that seek to violate user privacy. As it stands, there are existing e-ticketing applications which capture user movement patterns and divulge sensitive user information. The leaking of this information fosters attacks on the transportation system in the forms of Impersonation (unauthorized copying and use of tokens), Tracing (unauthorized monitoring of users) and Denial of Service (unauthorized blocking of legitimate users). Although there are some approaches to authenticating e-tickets and enforcing privacy, there is no mention of any solution that explicitly addresses the problem of inadequate user privacy. However, a solution was presented based on existing cryptographic tools and current RFID technology that is based on RFID tokens that are at most capable of performing symmetric cryptography [8].

From the literature review so far presented, it is clear that RFID Technology have gained favor in most industries where it is utilized to help streamlining several key processes that are needed to achieve optimal results. However, with the widespread use of RFID comes the issue of data privacy and security which if not carefully managed, may result in the technology being as troublesome as it is revolutionary. As a result, there is ongoing research aimed at developing robust protocols to govern how RFID components communicate, with added security and privacy features such as authentication and anonymity. Large scale deployment of these technologies, require careful planning to alleviate the challenges associated in order for the effort to be feasible. As it pertains to the application of RFID Technology in public transportation, there is a lot to be gained with regards to improved efficiency of transit systems that would benefit from reduced costs as a result of better decision making which in turn passes on an improved service to commuters. So with that as basis we will now look into our proposed research on dynamic bus trip scheduling and ticketing system using RFID and agent technology along with Android enabled mobile handset.

\section{INTELLIGENT AGENT BASED RFID BUS TRIP SCHEDULING SYSTEM}

The current public transportation system and its lack of dynamic trip scheduling capability along with the absence of a proper quantitative tool to effectively measure commuter crowding, has left weakened the operating inefficiently and the consequent loss. However, with the strategic introduction of RFID combined with Agent technology, these debilitating effects may be reversed. Before going into our proposed work in this direction, we first give a brief overview on RFID Tags, Reader and middleware association.

RFID technology consists of the following three components, a Tag, a Reader and the Middleware which interacts with the back-end database. The RFID Tag: consists of a microchip with data storage, limited logical functionality and an antenna which is tuned to receive radio frequency waves emitted by a reader or transceiver for allowing wireless transmission of data to the reader. For retail applications, the identifier takes the form of an Electronic Product Code (EPC). RFID scanners or readers usually consist of a radio frequency module, a control unit and a coupling element to interrogate the tags via radio frequency communication. Readers are usually connected through middleware to a back-end database. The RFID Middleware refers to specialty software that sits between the reader network and the application software to help process the significant amount of data generated by the reader network. Middleware is responsible for cleaning the data by eliminating false reads besides performing aggregation and filtering of data. Also, by monitoring multiple readers, middleware can detect the movement of RFID tags as they pass from the read range of one reader to another [4].

By using RFID enabled smart cards, RFID readers in buses can be made to get details on the number of. Persons who are on board a bus at any particular time by the scanning of passenger held smart cards. This information would then indicate the under utilization, optimum utilization or overloading of the service. Also the commuter at bus stops would key in from their android enabled smart phones, for the bus they are expecting for their intended destination. Additionally, the RFID reader affixed to a bus stop or traffic light will register the presence of a bus outfitted with an RFID tag. All these information would then enable agent based system to either inform the passenger of expected arrival time of bus or dynamically schedule the trip of a bus 
based on passenger requests and the passenger pattern, travel frequency and so on. Conversely, if an individual requiring special assistance for example a bus fitted with a wheelchair lift, he/she would then key in from his/her mobile at the bus stop, which could initiate a request to fast track such a bus to that location.

In addition to the commuter held smart cards interacting with RFID readers inside the bus, the buses themselves could be tracked via RFID tags and their approximate locations overlaid on a map such as Google Maps and made available by the bus company to mobile users for viewing on smart phones, tablets etc., through which bus schedule and route queries can be made. Passenger travel patterns could then be studied by mobile agents that would facilitate the fixing of bus trip schedules to passengers' mobiles based on travel requirements.

Lastly a cashless payment system may also be facilitated with the use of RFID technology, wherein upon boarding a bus, a passenger may pay for a ticket via his/her RFID enabled smart card. Here, the passenger simply waves the card within a few centimeters of an RFID reader installed in the bus and has his/her account debited and a ticket issued. In the event of the passengers' account approaching a low-balance, a top-up reminder may be also sent to his/her mobile. Along with each smart card tag, information about the holder of the smart card will also be provided. This could prove useful if an individual has to be identified for whatever reason later. For example, in the case of vandalism of buses, a camera may capture the act but if the individual cannot be identified visually, then there may be a chance to capture the person's identity if he or she is the holder of a RFID smart card.

\section{ARChitecture of INTELLIGENT AGENT RFID TECHNIQUE FOR BUS TRIP SCHEDULING}

For the proposed research work, we now give the architecture as shown in Fig. 1

The architecture shows that a commuter possessing RFID inside a bus and the information sent to an agent based system of Bus Company. In addition location of the bus at a traffic stop light intersection and at bus stop also detected by the RFID fitted in the bus and these information sent to the agent based system which facilitates users possessing mobile to query the location and schedule of the bus and view it on GMAP on their mobile. In addition commuters at bus stop can request for a bus by keying in from his/her mobile which the agent based system of bus company receives and accordingly expected arrival time of bus is sent to the mobile handset of the user or bus trip is scheduled dynamically based on number of requests for the bus, passenger pattern from past history, number of passenger information from RFID reader of bus, etc. The estimated time of arrival for buses will be derived from historical data gathered on buses operating in a particular area focusing on the various schedules, the estimated time to travel between stops or termination points, traffic congestion patterns and the current position of the buses with respect to latitude and longitude. All these communications from RFID tags and mobile devices of users to the agent based system happens wirelessly though wireless network.

Now based on the schematic shown in Fig.2, we here describe the functioning of bus/commuter tracking by RFID technology towards scheduling of bus by means of Data Flow diagram. We have shown in Fig. 1 that commuters and buses can be tracked by RFID reader and accordingly a bus can be scheduled. We now show in Fig. 2 as how when RFID tag is detected, data is captured by the agent based system for scheduling of buses. The system functioning be explained now.

The agent based system includes RFID data management facility system which is essentially a RFID data server containing RFID event handler towards logging the events triggered by bus at check-points like stop lights and bus stops and also commuter swiping their RFID enabled smart card. Also Expected Time of arrival of bus calculations will be performed by the RFID Data Management System and relayed to the mobile user's handset, which itself will be responsible for requesting and displaying the Gmap component along with the different Gmap features for map manipulation. This information about RFID event logging is actually stored in RFID event database pertaining to passengers and Buses. In addition details on commuters possessing RFID enabled smart card is stored in Smart card/commuter database which is checked for authenticity when swiping for boarding the bus or keying from mobile for requesting for bus and accordingly the event logged in RFID event database.

Bus, Route and Bus Schedule databases are interlinked as they are dependent on each other one way or the other. Bus database would contain the RFID identification of bus towards querying for trip schedule and route. Route and bus schedule database gives information to user on route and schedule of bus which is based on location information received from RFID reader fitted at bus stops and traffic stop light. This information is displayed by the agent to user's mobile handset on their GMAP We now would show the sequence of activities involved in the proposed system by means of a Sequence diagram shown in Fig.3.

In the sequence diagram shown, passenger boarding and exiting the bus possessing RFID enabled smart card are logged in the RFID event database. As passenger board the bus possessing RFID smart card, RFID reader fitted in bus reads it and sends to RFID data management system which logs the event and accordingly verified for authenticity by checking with commuter database. Once verified, the information stored in RFID event database along with location, date and time. The same applied towards passenger exiting bus. This information can be used if need be towards any vandalism or activity happened in bus for verification. Now that the passenger would use his Smart card which got credit to pay and get the ticket against RFID ticketing machine in bus. Once ticket issued automatically credit amount gets deducted and the same is updated in RFID event database. This sequence is shown in Fig.4.

There could be a situation where commuter standing at bus stop wants to know the schedule of bus he is waiting for and expected arrival time. The commuter uses the mobile application for requesting bus schedule from database. The bus schedule retrieved and displayed to commuter's mobile 
as shown in Fig. 5 In addition the commuter would also be able to view the current location of bus too from GMAP feature of mobile along with expected arrival time as shown in Fig. 6

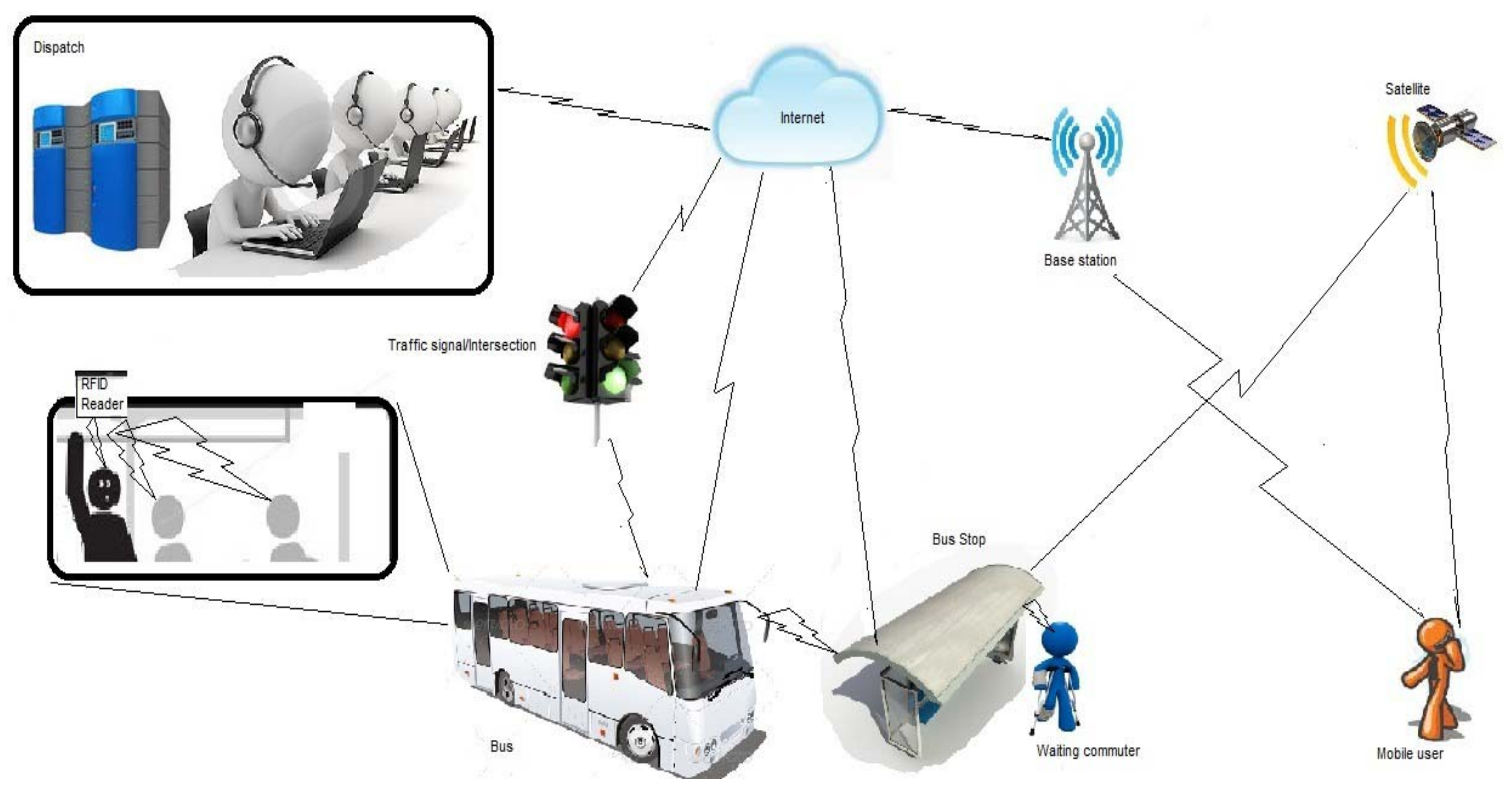

Fig. 1. Architecture of agent based RFID bus scheduling system

\section{Data Flow Diagram for bus/commuter tracking} using RFID
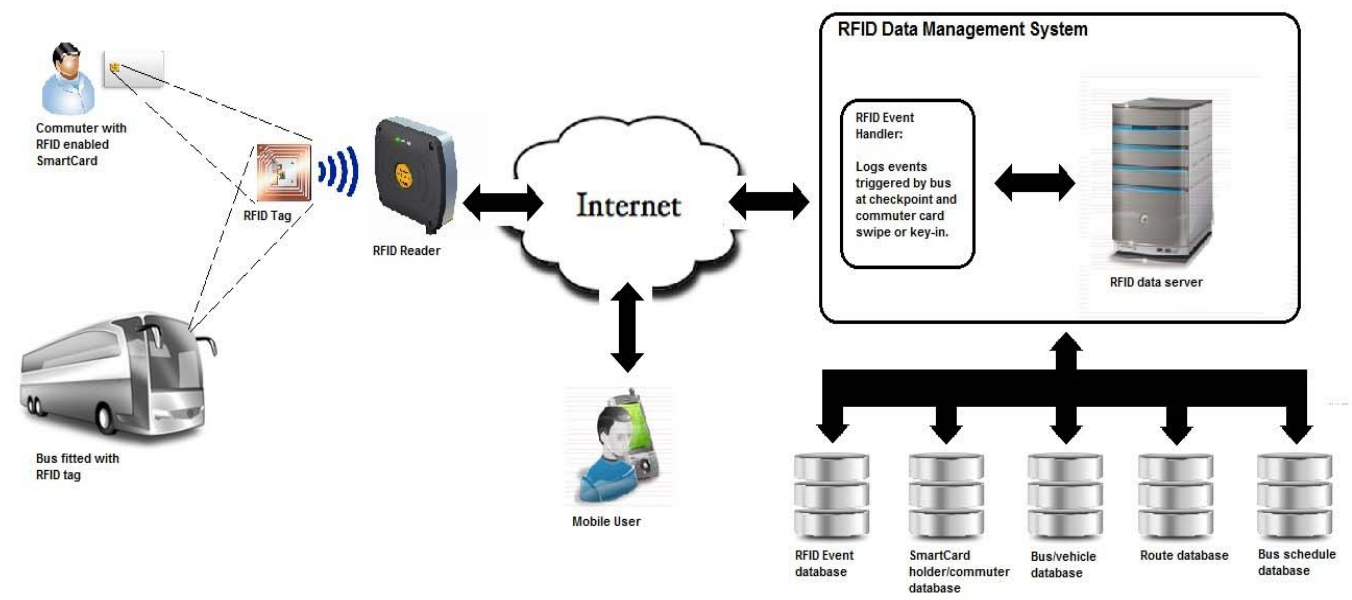

Fig. 2. Data flow diagram of bus/commuter tracking

Now in addition to passengers requesting for bus schedule and viewing via GMAP, there could be situations where lot of commuters are getting crowded in bus stops and requesting for particular bus for a certain location. So, in this case, instead of waiting for the scheduled bus at the expected time, commuters can use their mobile and key in with RFID enabled smart card for particular route bus as needed. Now based on the number of such requests received, the bus location, passengers on board and the passenger pattern from past history, a new route bus for the intended destination would be scheduled dynamically by the agent for the requesting commuters at the earliest. The sequence of information now flows from user's mobile is shown in Fig. 7.

Now similar to commuters requesting for buses at bus stops, it is also possible in a similar way for people with special needs to request for a bus supporting the special needs. This is not based on number of requests like in the previous case but once commuter with special needs keys in with
RFID smart card or national identification number from mobile for such a type bus, bus request sent to agent based system which would look into Bus vehicle database for such type buses availability and to immediately dispatch the bus to the commuter's location. This information flow from the displayed to user's mobile is as shown in Fig. 8

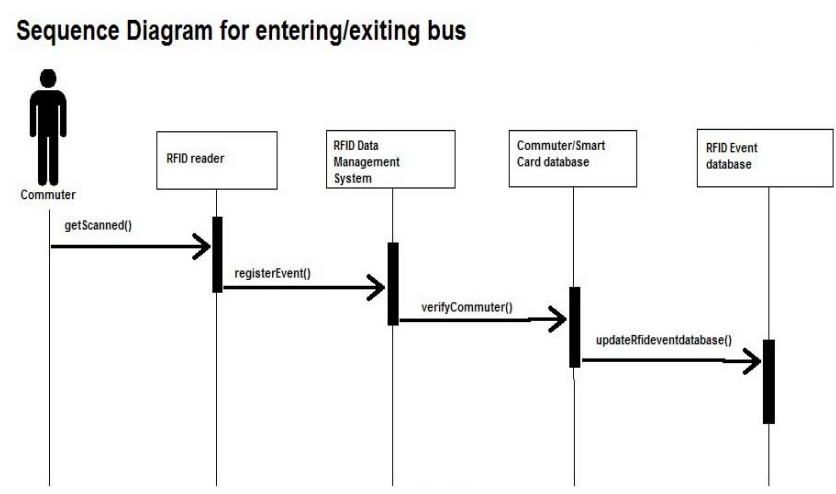

Fig. 3. Sequence diagram for boarding/exiting bus 
Sequence Diagram for paying fare via SmartCard

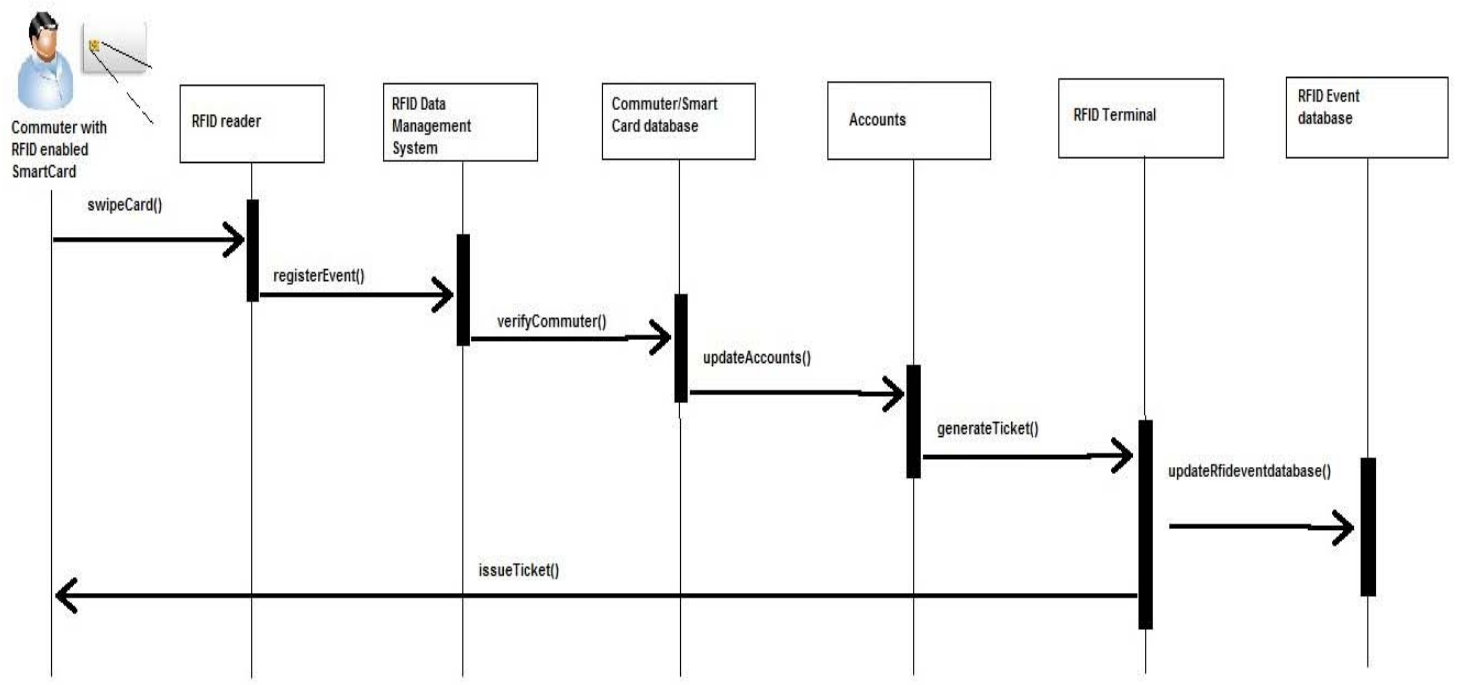

Fig. 4. Sequence diagram for paying ticket

\section{Sequence Diagram for checking bus schedule via mobile}

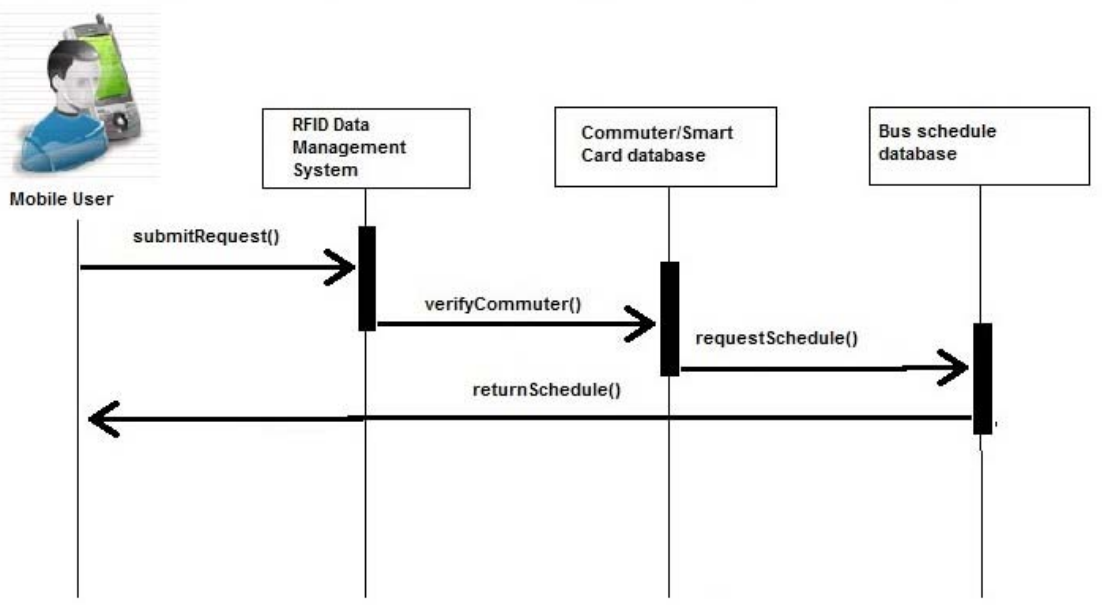

Fig. 5. Sequence diagram for bus schedule

Sequence Diagram for checking on available buses and their location on Gmap via mobile

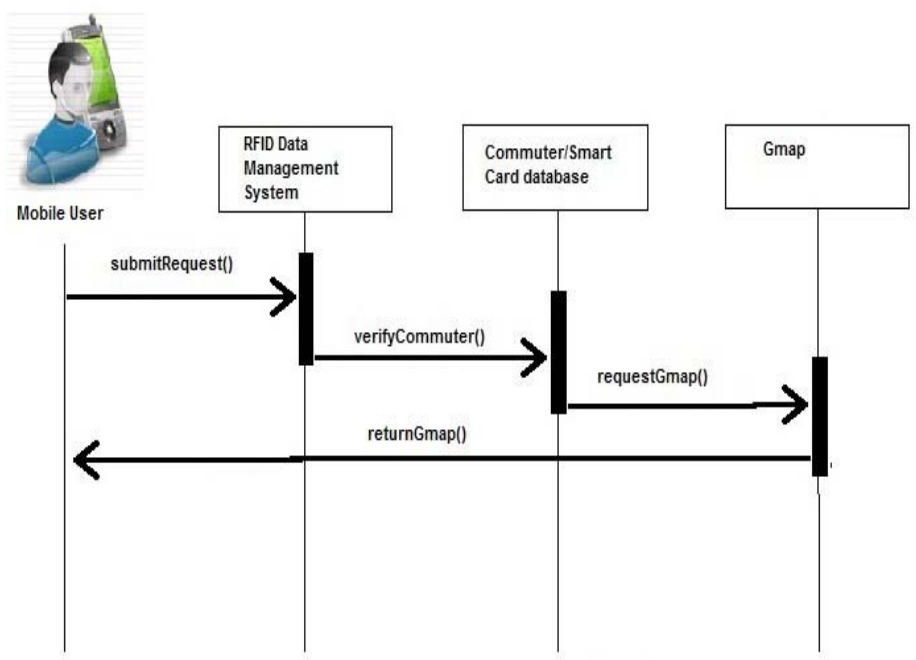

Fig. 6. Sequence diagram for bus with gmap location 


\section{Sequence Diagram for commuter keying-in at bus stop via mobile}

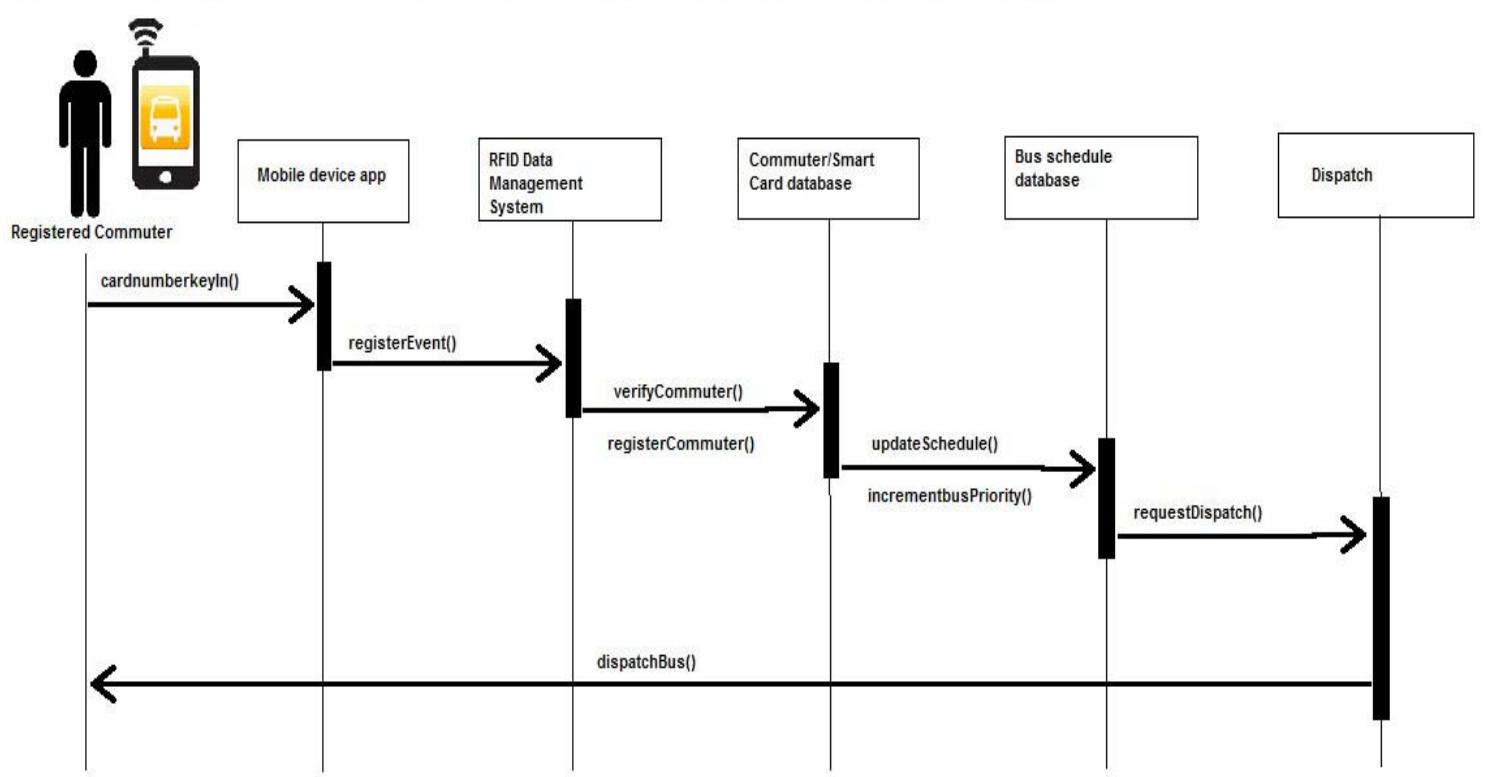

Fig. 7. Sequence diagram for commuter key in at bus stop from mobile

\section{Sequence Diagram for keying-in and requesting bus via mobile for commuters with special needs}

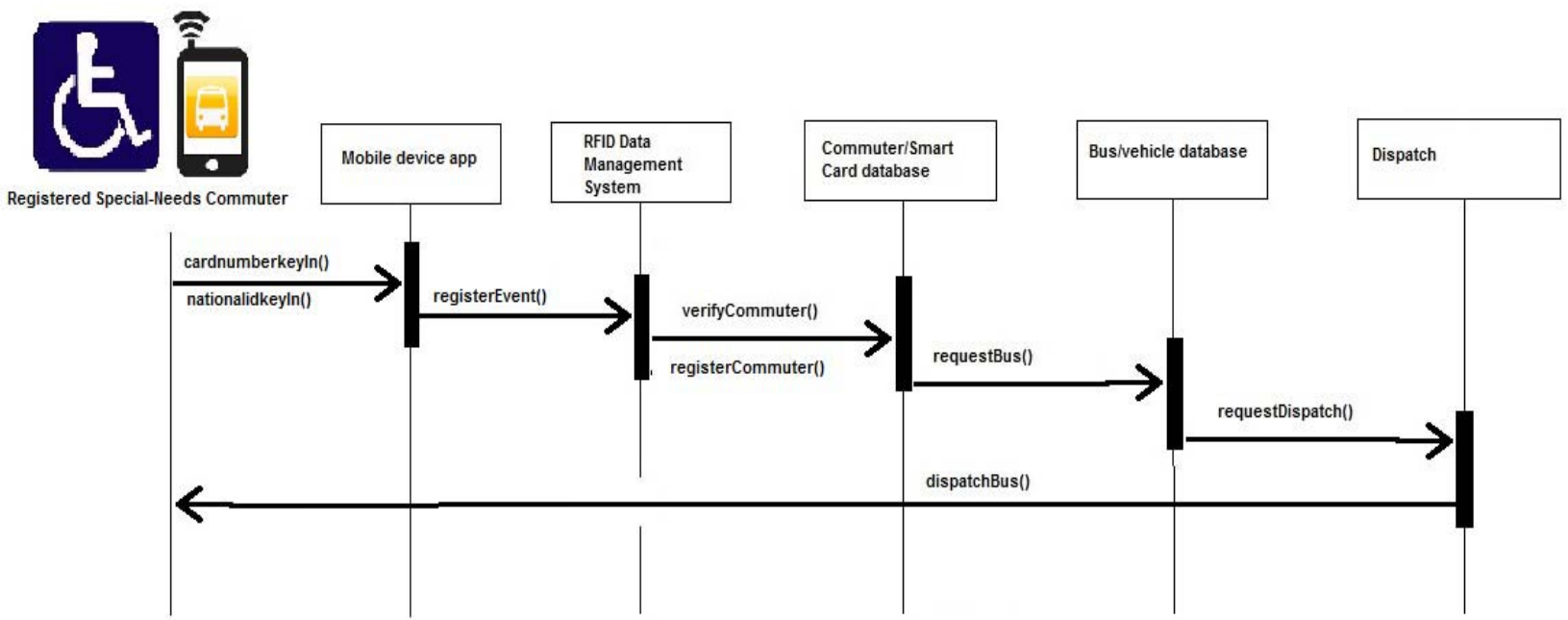

Fig. 8. Sequence diagram for special needs of a commuter

\section{CONCLUSION}

In conclusion, for commuters facing challenges as far as accessing public transportation as needed is mostly attributed to the static scheduling of buses, which more often than not seeing buses operating within the same pattern, regardless of fluctuations in commuter demand. Quite amount of research has been carried out in using Radio Frequency Identification (RFID) technology in public transportation system towards tracking of passengers when they board and exit, tracking of bus by deploying at traffic stop lights, intersection etc for updating bus arrival time which user can view form his cell phone. In addition research has also been conducted in using mobile agents with RFID towards passenger demand and carrying capacity.

So based on the research work reported so far, we here propose an agent based system towards dynamic trip scheduling of buses by means of passenger tracking who more or less follow a specific pattern, which also would be noted and included in forward planning by means of intelligent agents. In addition commuters requesting buses with special needs/facility and smart card way of paying ticket, have been proposed. Lastly commuters be tracked possessing RFID based smart card while boarding and exiting bus for future purpose in case of any illegal activity. The above research work has been reported in the paper in form of architecture, Data Flow diagram and Sequence diagrams. We are now carrying out the implementation of the proposed system using Android and JADE-LEAP agent development kit.

\section{REFERENCES}

[1] X. L. MA, Y. H. Wang, F. Chen, and J. F. Liu, "Transit smart card data mining for passenger origin information extraction," Journal of Zhejiang University-SCIENCE C (Computers \& Electronics), vol. 13 no. 10, pp. 750-760, 2012. 
[2] C. Oberi and T. T. Miguel, "Performance evaluation of UHF RFID technologies for real-time passenger recognition in intelligent public transportation systems," IEEE Transactions on Intelligent Transportation Systems, vol. 11, no. 3, pp.748-753, 2010.

[3] D. C. Karaiskos, P. Kourouthanassis, and G. M. Giaglis, "User acceptance of pervasive information systems: evaluating an RFID ticketing system," in Proceedings of ECIS, Athens, Greece, 2007.

[4] B. Menzes, K. Laddhad, and B. Karthik. Challenges in RFID deployment - a case study in public transportation. [Online]. Available: http://www.it.iitb.ac.in/ kamlesh/Page/Reports/iceg06.pdf

[5] J. L. Wang, "Privacy and ethical issues in location-based tracking systems," in Proceedings of IEEE International Symposium on Technology and Society, Illinois, USA, 2009.

[6] S. Thomas, H-J, Chae, B. Defend, and K. Fu, "Privacy for public transportation," in Proceedings of Privacy Enhancing Technologies Workshop, Cambridge, UK, 2006.

[7] P. Bouman, M. Lovric, E. V. D. Hurk, L. Kroon, and P. Vervest. Recognizing demand patterns from smart card data for agent-based micro-simulation of public transport. [Online]. Available: http://www.ia.urjc.es/att2012/papers/att2012_submission_21.pdf

[8] A-R .Sadeghi, I. Visconti, and C. Wachsmann. User privacy in transport systems based on RFID e-tickets. [Online]. Available: http://www.trust.rub.de/media/trust/publications/SaViWa08.pdf

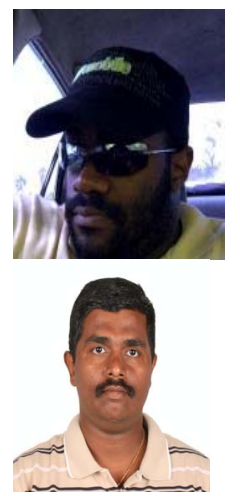

Paul Hamilton is currently Graduate student pursuing M.sc computer Science in department of computing, University of West Indies, Jamaica since 2011. He hold Bachelor's degree in computing from University of West Indies in 2011. amaica, West Indies. He has supervised around 30 research students leading to M.Sc, ME, M.Phil and M.S degrees and currently supervising students leading to M.sc, M.phil and Ph.d respectively. He has got to his credit, as on date, about 50 fully refereed research papers published in the Proceedings of major IEEE international conferences, as Book Chapters and in International Journals.. His current research interests are mainly towards Wireless Sensor Networks, Mobile Commerce, Intelligent Agents' used in the Health, Commercial and Engineering sectors. 